Cite this: J. Mater. Chem. C, 2014, 2 , 349

Received 7th October 2013

Accepted 28th October 2013

DOI: $10.1039 / \mathrm{c} 3 \mathrm{tc} 31969 \mathrm{e}$

www.rsc.org/MaterialsC

\section{Down-scaling of resistive switching to nanoscale using porous anodic alumina membranes $\dagger$}

\author{
Jakub Kolar, ${ }^{a}$ Jan M. Macak, ${ }^{\star a}$ Kazuya Terabe ${ }^{b}$ and Tomas Wagner ${ }^{a}$ \\ An advanced approach for resistive switching memory cells based on porous anodic alumina $\left(\mathrm{Al}_{2} \mathrm{O}_{3}\right)$ \\ membranes is reported. The effective resistive switching resulting in 6 orders of magnitude difference in \\ resistivity between "on" and "off" states of the cell is achieved by specific electronic and ionic interaction \\ between $\mathrm{Ag}$ nanowires filled in the membrane and an ionic conductor $\left(\mathrm{Ag}_{x} \mathrm{AsS}_{2}\right)$ deposited on the \\ membrane by thermal evaporation. This easy and robust approach can be exploited for deposition of \\ other ionic conductors for novel types of memories.
}

\section{Introduction}

Current electronic devices (including mobile phones, tablets, and cameras) require increasingly more powerful batteries and memories for their operation. Based on the market demand, there has been for several past years a great attention given to the exploration of new data storage approaches that would enable an increase in the capacity and the speed of memories. Nowadays, conventional data storage technologies, based on charge storage (e.g., DRAM, Flash), are close to their limits. ${ }^{1}$ Therefore, there is an urgent need for development of new memory devices based on novel concepts. Several feasible data storage approaches have recently been explored, such as spinbased, ${ }^{2}$ phase change ${ }^{3}$ and resistive switching ${ }^{4}$ memories. The resistive switching memories, whose differences between the resistivity states enable storage of information, are based on several different mechanisms: thermal, electronic and ionic effects. ${ }^{4}$ In particular, cells based on the ionic effect currently offer intriguing possibilities for more sophisticated data storage applications, such as memristors ${ }^{5}$ or brain like computation. ${ }^{6}$ Moreover, recently published data on these memories seem to be very promising: switching time shorter than $35 \mathrm{~ns},{ }^{7}$ smooth operation for more than $10^{11}$ cycles, $^{8}$ and very low energy consumption. ${ }^{9}$

Such performances stem essentially from nanoscale operations. Thus, memories and their parts - cells - have to feature essentially all their dimensions on the nanoscale. Although it is already clear that scale reduction of all memory cell constituents towards the nanoscale is a step forward for memories with

${ }^{a}$ Department of General and Inorganic Chemistry, Faculty of Chemical Technology, University of Pardubice, Nam. Cs. Legii 565, 53002 Pardubice, Czech Republic. E-mail: jan.macak@upce.cz; Tel: +420466037401

${ }^{b}$ International Center for Materials Nanoarchitectonics (WPI-MANA), National Institute for Materials Science, 1-1 Namiki, Tsukuba, Ibaraki, 305-0044, Japan

$\dagger$ Electronic supplementary information (ESI) available: SEM characterization and details on electrical measurements. See DOI: 10.1039/c3tc31969e improved performances, the exact switching mechanism in the cell is not yet well understood and has to be further investigated. This is in particular true for memories and their cells based on the ionic switching, where either cations ${ }^{\mathbf{8}}$ or anions ${ }^{\mathbf{1 0}}$ play a crucial role in the switching process. Some hurdles in understanding the mechanism may arise from the fact that obtaining very small cell parts with a very high precision, closely confined and contamination-free, is not an easy task. As a matter of these facts, thin-film deposition techniques have been employed to prepare these cells. Thermal evaporation, ${ }^{7}$ magnetron sputtering, ${ }^{11}$ or laser ablation ${ }^{12}$ can create homogeneous thin films, whose thickness can be controlled. On the other hand the control of other two dimensions (width and length) remains difficult. As alternatives, common lithographical techniques, such as photolithography, ${ }^{13} \mathrm{UV}$ nanoimprint lithography ${ }^{\mathbf{1 4}}$ and electron beam lithography, ${ }^{7}$ have been employed. However, these techniques have also their limits in the resolution of the features and may lead to mechanical delamination of cell layers and contamination from the solvents, resins, etc.

In this work we demonstrate an advanced approach for the construction of memory cells based on porous anodic alumina membranes (further denoted as AAO) filled with $\mathrm{Ag}$ nanowires and covered by a layer of ionic conductor (amorphous chalcogenide glass), where essentially a number of nanowires interfaced with the ionic conductor is responsible for the switching performance providing a sum of individual local contributions of their electrical fields upon switching. In this approach, the AAO membrane with long channels ( $\mu \mathrm{m}$ scale) created by small pores (nm scale) acts as a matrix for a large number of electrodeposited $\mathrm{Ag}$ nanowires filled homogeneously over the membrane. After the ionic conductor has been deposited on the top of such AAO membrane, the as-filled Ag nanowires become connected with it. Thus, at their interface many small memory electrodes are formed that are actively involved in the switching process and have specific switching characteristics. This very 
effective, yet simple approach is significantly different from memory cells based on stacked multilayers, ${ }^{11}$ because here instead of forming purely layered electrode systems, we create a huge number of local nanosized electrodes. In general, AAO membranes have offered intriguing possibilities for synthesis of various template-assisted nanowires of metals, ${ }^{15,16}$ polymers, ${ }^{17}$ semiconductors ${ }^{18}$ and advanced nanostructured systems. ${ }^{19,20}$ In a previous study of one of the coauthors ( $\mathrm{K}$. Terabe), AAO membranes have been used for construction of crystalline $\mathrm{Ag}_{2} \mathrm{~S}$ based resistive memory cells, which were prepared by sulphurization of the $\mathrm{Ag}$ layer. ${ }^{21}$ However, in that work the thickness of $\mathrm{Ag}_{2} \mathrm{~S}$ was approximately $5 \mu \mathrm{m}$, which is too thick to explore nanoscale interfaces used in the present work. There have been other studies focusing on the use of single nanowire switching cells $^{22}$ and exploration of various metal nanowires often with even a narrower diameter than in this work. ${ }^{23,24}$ However, the exploration of thin layers of an amorphous ionic conductor present on an AAO membrane as key components of the resistive memory cells has never been reported. Compared to previous literature, ${ }^{21}$ this approach can be used for a range of ionic conductors. As a case example, we selected amorphous chalcogenide $\mathrm{AsS}_{2}$, since it can readily accept $\mathrm{Ag}$ ions by photoinduced diffusion and dissolution, and electromigration and thus a variety of compositions can be prepared..$^{25}$

For clarity of this paper and in particular for the discussion of the results, we want to firstly introduce and briefly discuss the working principle of the memory cell based on the cationic resistive switching, where the metal dissolution in the ionic conductor is the key process in this memory type. ${ }^{21,26}$ As shown in Fig. 1a, the cell consists of distinct layers of diffusive metal (Ag), ion conducting material (Ag-photodoped $\mathrm{AsS}_{2}$ ) and nondiffusive metal as a contact $(\mathrm{Al})$. When a positive bias is applied on the diffusive metal (as depicted in Fig. 1b), the metal undergoes oxidation and its cations are dissolved in the ion conducting material, through which they further migrate towards the cathode (point I). At a certain breakpoint the cations are reduced on the cathode (made of $\mathrm{Al}$ ) in such a quantity that they form a metallic filament through the ion conducting material (point II). Thus both the electrodes become connected by the metallic filament and the cell resistivity rapidly decreases. On the other hand, applying a negative bias causes dissolution of the conducting filament, while the overall resistivity remains unchanged for some time. Right after
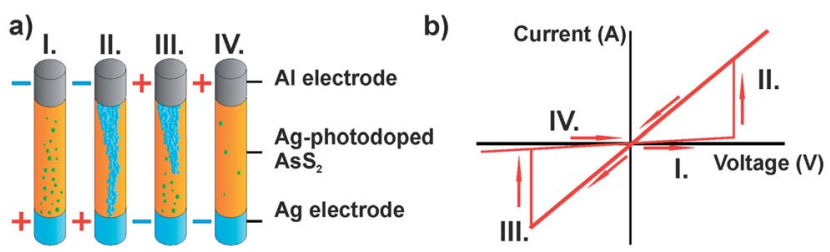

Fig. 1 (a) The scheme of operation of a memory cell based on ionic resistive switching, thicknesses of all layers are on the order of hundreds of nanometers. Symbols + and - represent applied polarities during each particular stage. Green and blue points represent $\mathrm{Ag}^{+}$and $\mathrm{Ag}^{0}$ species, respectively. (b) The scheme of 4 stages through which the cell passes within each cycle of operation. another breakpoint (point III) the overall resistivity of the cell increases again, till its original value (point IV). This can essentially be repeated for many times, until the ionic conductor layer is capable of forming/dissolving the filament accompanied by large resistivity difference. Typically, the cell lifetime is limited by the rupture of the layered cell structure, which may be caused by the heat generation and/or mass flow. Therefore, the potential window has to be carefully selected to maintain flowing current at such a level to maintain long cell lifetime (represented by a large number of cycles without damage). The discussion about the filament built-up will be provided later in the Results and discussion section.

\section{Experimental}

Fig. 2 shows in detail two types of memory cells used in the present study. Their electrochemical switching processes follow all particular stages in the switching scheme (Fig. 1), but their interlayer construction is different. The first cell type (Fig. 2a) is based on the AAO membrane filled with $\mathrm{Ag}$ nanowires (further denoted as CELL AAO). For these samples, we used commercially available $50 \mu \mathrm{m}$-thick Whatman AAO membranes with open pores on both sides (with an average pore diameter of approximately $220 \mathrm{~nm}$ ). The surface roughness of such membranes is up to $1 \mu \mathrm{m}$ due to grains and their boundaries. Subsequently, we covered always one side of the membrane by an evaporated $\mathrm{Ag}$ layer (300 $\mathrm{nm}$ thick) that served as a cathode during $\mathrm{Ag}$ electrochemical infilling of AAO. The filling of membranes stems from procedures described in the literature. ${ }^{27}$ In brief, pulse deposition was carried out in electrolytes consisting of $45 \mathrm{~g} \mathrm{l}^{-1} \mathrm{AgNO}_{3}, 77 \mathrm{~g} \mathrm{l}^{-1} \mathrm{CH}_{3} \mathrm{COONH}_{4}, 32 \mathrm{ml} \mathrm{l}^{-1}$ $\mathrm{NH}_{3} \cdot \mathrm{H}_{2} \mathrm{O}$ and a small amount of $\mathrm{NaOH}$ to adjust the $\mathrm{pH}$ to $\approx 9$. The Ag bottom layer of AAO served as the cathode and Pt plate served as the anode. A pulse generator (Agilent 8110A $150 \mathrm{MHz}$ Pulse Generator) was set to repeat $10 \mathrm{~ms}$ pulse every $0.5 \mathrm{~s}$. The resulting AAO structures had nearly $100 \%$ of the pores filled by Ag. However, their surfaces were covered by Ag overgrown of the pores and some pores were not filled completely (see ESI, Fig. $\mathrm{S} 1 \dagger)$. To remove the overgrown layer and to achieve such depth of AAO membranes, where all pores are filled, the Ag-filled AAO membranes glued to a $\mathrm{Cu}$ plate (using Ag-glue) were ground using SiC emery paper (with grid 2500) down to
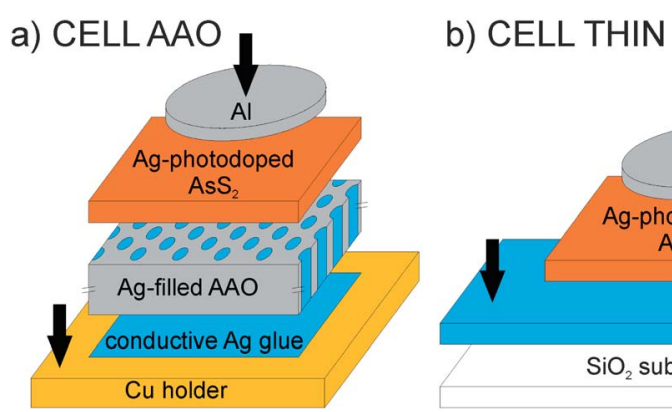

Fig. 2 (a) Scheme of CELL AAO and (b) scheme of CELL THIN. Arrows indicate contacting points. 
$25 \mu \mathrm{m}$ and polished in order to achieve a smooth surface (roughness $\approx 10 \mathrm{~nm}$ ), perpendicular to the direction of $\mathrm{Ag}$ nanowires.

The second cell type is based on multilayers of thin films of same components, but without the AAO membrane. For these cells (in further text denoted as CELL THIN), we used silica glass as the starting substrate that was coated by Ag through evaporation resulting in an approx. $300 \mathrm{~nm}$ thick layer.

Next steps were identical for both cell types. In brief, both structures were covered by approx. $100 \mathrm{~nm}$ thin films of $\mathrm{AsS}_{2}$ using the thermal evaporation technique. Insulating $\mathrm{AsS}_{2}$ was modified into ion conducting $\mathrm{Ag}_{x} \mathrm{AsS}_{2}$ by photo-induced diffusion of $\mathrm{Ag}$. At the end, $\mathrm{Al}$ non-diffusive electrodes (approx. $200 \mathrm{~nm}$ in thickness, $1 \mathrm{~mm}^{2}$ surface area) were evaporated on cell surfaces by thermal evaporation of Al through an appropriate mask. Amorphous $\mathrm{AsS}_{2}$ chalcogenide glass ${ }^{28}$ was selected as a model ionic conductor from the great family of ionic conductors, including oxides, ${ }^{10}$ polymers, ${ }^{29}$ amorphous $^{30}$ and crystalline chalcogenides. ${ }^{31}$ Amorphous glassy chalcogenides feature themselves by simple preparation in bulk and also in thin film form, ${ }^{32}$ and by tuneable properties as ion/electron conductivity ratio, ${ }^{28}$ content of chalcogen,$^{33}$ chemical stability ${ }^{34}$ and phase separation. ${ }^{35}$ In contrast to the previous work, ${ }^{21}$ the ionic conductor was purely amorphous here. Since $\mathrm{AsS}_{2}$ itself is a very good insulator, it was photodoped by $\mathrm{Ag}$ (ref. 36) in order to increase its ionic conductivity by light emitted from a mercury lamp for 30 minutes under pure $\mathrm{N}_{2}$ (purity $4 \mathrm{~N}$ ) atmosphere. Ag electrodes served as the source of $\mathrm{Ag}$ for this photo-diffusion process.

Finally, Al electrodes were deposited by thermal evaporation of $\mathrm{Al}$ on the $\mathrm{Ag}$-photodoped $\mathrm{AsS}_{2}$. A metallic (Al) mask was used in order to achieve circular electrodes with a diameter of $1 \mathrm{~mm}^{2}$. All necessary steps were optimized and the real structure after each step was carefully observed using a scanning electron microscope (Mira3 XM FEG SEM, Tescan). All in all, these procedures resulted in two types of principally very similar cell types, but having strongly different design and dimensions of the $\mathrm{Ag}$ diffusive electrode.

\section{Results and discussion}

Fig. 3 shows SEM images of an empty AAO membrane and an Ag-filled AAO membrane as an example of CELL AAO. For comparison, we provide top-views of the empty membrane (Fig. 3a) and the polished Ag-filled AAO membrane (Fig. 3b). From these images one can see that after polishing the surface becomes very uniform and smooth. Moreover, from Fig. 3c and $\mathrm{d}$ that display the whole cell in the cross-sectional view, it becomes clear that the original membrane thickness of $50 \mu \mathrm{m}$ was ground down to approximately $25 \mu \mathrm{m}$ and polished, resulting in a smooth membrane having continuous embedded nanowires in the pores. The membrane thinning to approximately half of its original thickness is responsible for the fact that the original pore diameter at the membrane top decreased from approximately $220 \mathrm{~nm}$ to approximately $190 \mathrm{~nm}$. Moreover, Fig. 3e and f show the same layers in cross-sectional views under higher magnifications, where contrast difference

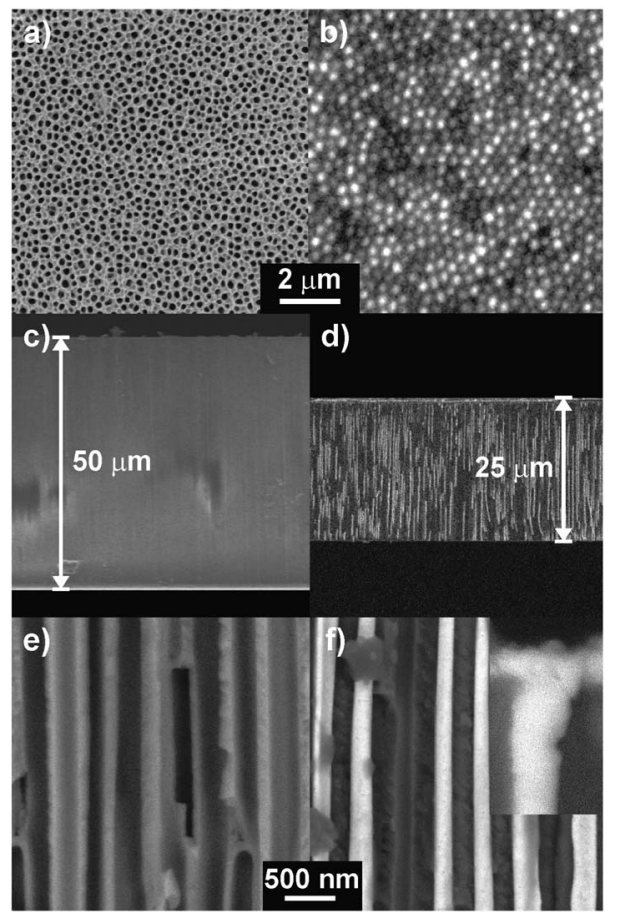

Fig. 3 SEM images of CELL AAO. Left column displays images of an empty AAO membrane, right column displays images of a polished Agfilled AAO membrane. We show top-views ( $a$ and b), cross-sectional views of the membrane ( $c$ and $d$ ) and high magnifications of the same (e and f). The inset of (f) shows the interface between Ag-filled AAO and $\mathrm{Ag}_{x} \mathrm{AsS}_{2}$ after switching experiments have been performed (10 cycles).

between AAO and Ag (owing to different quantities of backscattered electrons) reveals homogeneous filling of AAO pores by $\mathrm{Ag}$ nanowires. Finally, the inset of Fig. $3 \mathrm{f}$ shows a detailed cross-sectional view of the interface between $\mathrm{Ag}$-filled AAO and $\mathrm{Ag}_{x} \mathrm{AsS}_{2}$ after switching experiments have been performed (10 cycles).

As one can see from the white appearance (employing signal from the back-scattered electron detector), resulting $\mathrm{Ag}_{x} \mathrm{AsS}_{2}$ contains quite a significant amount of Ag. However, its exact amount cannot be measured accurately by surface analytical techniques (such as EDX or XPS), because these techniques enhance Ag migration.

Cell designs used in the present study allow us to investigate the effect of the size and shape of the $\mathrm{Ag}$ electrode on the resistive switching behavior. Therefore, the as-prepared samples were subjected to cyclic voltammetry. Fig. 4 shows comparison of voltammograms recorded for samples of CELL AAO (Fig. 4a) and CELL THIN (Fig. 4b). A detailed description of these measurements is given in the ESI. $\dagger$ For the purpose of clarity, we provide response in terms of current (left axes) as well as current densities (right axes). Both plots show data recorded within four measurement cycles: $1^{\mathrm{st}}, 5^{\mathrm{th}}, 10^{\text {th }}$ and $20^{\text {th }}$, all of them reflect typical switching behavior as described in Fig. 1. For both cell types, the switch "on" and switch "off" occur abruptly and the threshold voltages are below $0.3 \mathrm{~V}$ and $-0.3 \mathrm{~V}$, respectively. The obvious differences in current levels and shifts 
a) CELL AAO

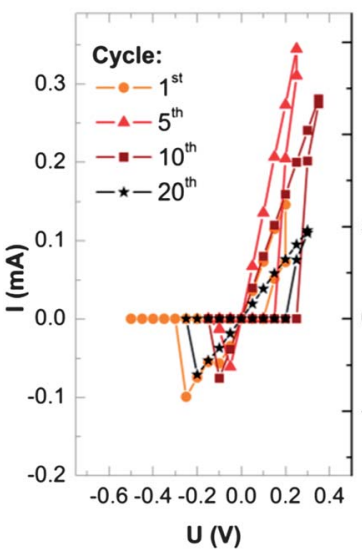

b) CELL THIN

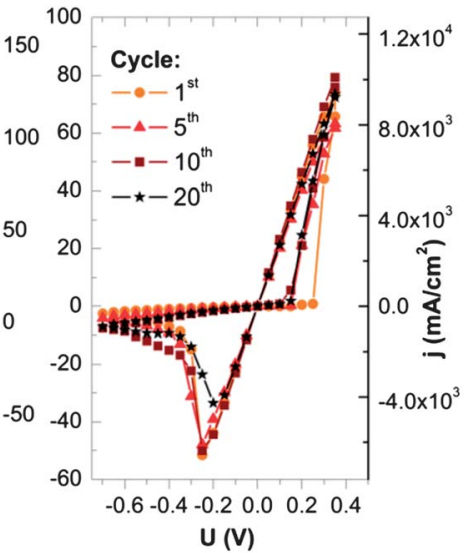

Fig. 4 Cyclic voltammograms of (a) CELL AAO and (b) CELL THIN for several particular cycles. Left $Y$-axes display currents and right $Y$-axes display current densities.

of curves upon cycling stem partially from the difference in contact areas on samples during switching, but mainly from the changing stoichiometry. The contact area for CELL AAO is approximately $30 \%$ of the contact area of the CELL THIN (as it was calculated from the porosity of AAO assuming that all its pores are filled with $\mathrm{Ag}$, which is almost the case after polishing as demonstrated in Fig. 3). The differences in performance shown in Fig. 4 originating from changing stoichiometry will be discussed later. The data presented in Fig. 4 are sufficient for the demonstration of the switching of the cells. However, they do not provide straightforward information about cyclability and differences in resistivities upon extended cycling.

Therefore, recorded voltammetric data were further analyzed (details are given in the ESI $\dagger$ ) in order to point out the resistivity of the "on" and "off" states in each cycle. Results are plotted in Fig. 5 that shows the "on" and "off" resistivity for both cell types and their evolution upon cycling. In addition resistivities of studied cells before the first switch "on" are also presented, so the overall history of the sample is recorded.

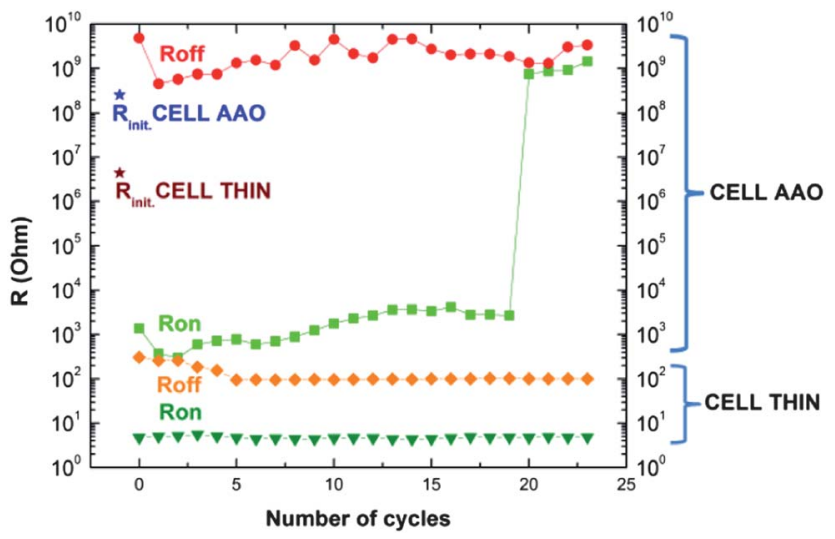

Fig. 5 Comparison of resistance data between the CELL AAO and the CELL THIN.
From Fig. 5 a huge change in "on" and "off" state resistivities for CELL AAO in comparison with CELL THIN is clearly visible. In the case of CELL THIN resistivities were much lower (the "on" state resistivity was below $10^{1} \Omega$ and the "off" state resistivity was above $10^{2} \Omega$ ) for all measured cycles. It is also noteworthy that its initial resistivity $R_{\text {int }}$ was comparably high $\left(4.4 \times 10^{6} \Omega\right)$.

For CELL AAO resistivities were much higher, the "on" state resistivity was below $10^{4} \Omega$ and the "off" state resistivity was above $10^{8} \Omega$ for all measured cycles. The initial resistivity of the "on" state of the CELL AAO was $2.6 \times 10^{8} \Omega$, which is quite close to typical "off" state resistivity. However, in the $21^{\text {st }}$ cycle the resistivity of the "on" state of CELL AAO became quite high and the switching behavior was lost. This behavior was observed throughout all samples, usually after 20 and more cycles.

Taking into account our results and observed findings we can discuss now about the switching mechanism. In CELL THIN the initial resistivity $\left(R_{\text {int }}\right)$ is much higher than resistivities at the "off" state $\left(R_{\text {off }}\right)$, i.e. immediately within the first switching cycle the Ag stoichiometry in the ionic conductor significantly changes. The lower resistivity indicates that there is a significant uptake of $\mathrm{Ag}$ by electromigration into the ionic conductor that leads to a significant decrease of the overall resistivity. The reasons for such an uptake are essentially twofold.

At first, we can assume that the $\mathrm{Ag}$ rich area contains conductive $\mathrm{Ag}^{0}$ clusters or filaments, as observed experimentally by Guo et al. ${ }^{37}$ and schematically depicted in Fig. 1a. The filament has been shown in the literature to have most likely the dendrite shape. ${ }^{37}$ The shape and the number of dendrites, however, stem from the amount of dissolved metal in the ionic conductor. The dissolved amount is influenced by the initial metal amount and by the electric field set at a certain potential difference. The filament formation is naturally very desirable and it is the most important phenomenon for the whole cell. ${ }^{4}$ However, at the same time, it is also the most critical factor. If the created filament is too big, it cannot be completely dissolved and the resulting material will not reach its original resistivity. ${ }^{21}$

Secondly, a stoichiometric enrichment of the $\mathrm{Ag}$ content in $\mathrm{Ag}_{x} \mathrm{AsS}_{2}$ may occur that leads to a significant change of the intrinsic resistivity of the $\mathrm{Ag}_{x} \mathrm{AsS}_{2}{ }^{28,38}$ Naturally, both effects have an impact on decreased resistivity of the cell, no matter whether we speak about the "on" or the "off" state, as their difference is rather low in the case of CELL THIN. It has to be noted that for this cell type, the Ag comes from an almost infinite source of $\mathrm{Ag}$ layer and the presence of such rich source must influence the resulting distribution significantly. Without specifying the dominant effect, it is reasonable to assign better reproducibility among switching cycles observed during cycling voltammetry to the fact that the "on" and "off" states are accompanied by relatively minor changes in the layers of CELL THIN in terms of their stoichiometry, not strong enough to alter the electrochemical characteristics during cycling (Fig. 4b).

In contrast, the situation for CELL AAO is different in two points. Firstly, the electric field flowing through the cell is more focused (i.e. less randomly oriented), due to the regular alignment of the nanowire Ag-electrode embedded in the AAO matrix. This must have an impact on the creation of conducting pathways through the ionic conductor. Similar effects on 
electric field caused by nanosized structures have been described in the literature. ${ }^{39,40}$ The other main point is the limited source of $\mathrm{Ag}$ for photoinduced diffusion and filament creation compared to CELL THIN. In other words, there is not as much Ag available in the nanowires as in the bulk $\mathrm{Ag}$ layer due to the AAO porosity of $30 \%$. For instance, if we would compare the amount of $\mathrm{Ag}$ available for the switching between $300 \mathrm{~nm}$ thick Ag layer and $300 \mathrm{~nm}$ long Ag nanowires embedded in $300 \mathrm{~nm}$ thick AAO membrane, Ag nanowires would account only for $30 \%$ of the $\mathrm{Ag}$ content compared to the bulk $\mathrm{Ag}$ layer. However, the resistance of the "on" and "off" states in the CELL AAO is huge - almost 6 orders of magnitude.

In order to provide a more insight into the CELL AAO interface between the ionic conductor and $\mathrm{Ag}$ nanowires embedded in the AAO matrix, we show in Fig. 6 a comparison of images acquired by SEM before the entire switching experiment and after the entire switching experiment with the cell damaged. During the SEM screening, we intentionally used both types of detectors separately (secondary-electron detector, SE, and back-scattered electron, BSE, respectively) and simultaneously to evaluate the connection of $\mathrm{Ag}$ nanowires to the layer of ionic conductor in terms of morphology and composition.

As it can be clearly seen from the comparison, the $\mathrm{Ag}$ nanowires embedded in the AAO membrane are in solid contact with the ionic conductor before switching (i.e. after Ag photodiffusion into the ionic conductor) and naturally during the

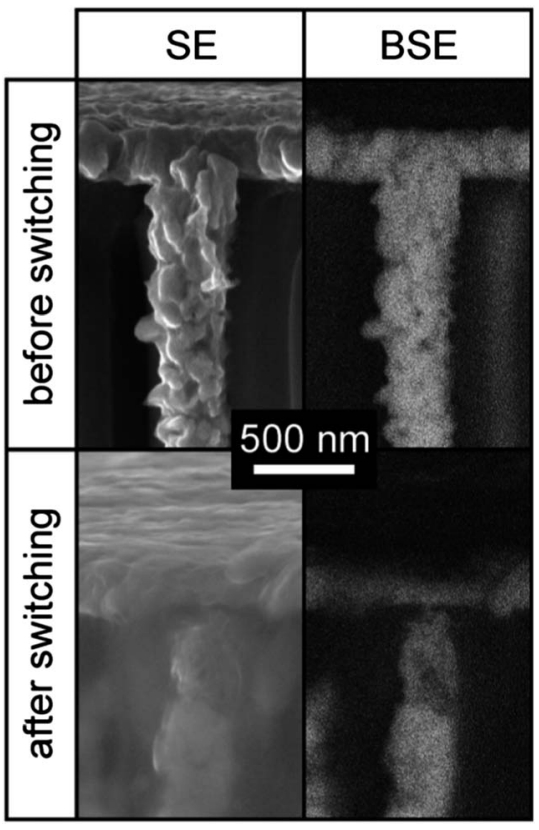

Fig. 6 SEM images of the CELL AAO sample showing the interface of the ionic conductor and Ag nanowires embedded in the AAO matrix. SE stands for imaging using a secondary-electron detector addressing the morphology of the observed samples, BSE stands for a backscattered electron detector addressing the compositional differences on the interface. Observations were done under a high magnification to see properly details of the interface (magnification 100 000×, working distance $5 \mathrm{~mm}$ ). The sample "after switching" was observed after recording 21 cycles and stopping the experiment afterwards. switching (as shown in Fig. 3f), while after a critical number of switching cycles (under given conditions used in this work typically between 20 and 25 cycles) they become disconnected as a result of $\mathrm{Ag}$ depletion. This is in particular apparent from the BSE image of the switched cell, where a clear gap between the Ag nanowire and the ionic conductor is visible. We have observed these features in all samples involved in our switching experiments. As a matter of fact, the resistive switching is accompanied by relatively strong $\mathrm{Ag}$ mass flow (leading to its depletion) on the nanoscale interface between Ag-filled AAO, ionic conductor and $\mathrm{Al}$ electrode.

Moreover, this explains the shift between cycles recorded for CELL AAO (Fig. 4a), in particular for the CELL AAO resistance in the "on" state (see $R_{\text {on }}$ for CELL AAO in Fig. 5) that shows an overall increase during the switching. This increase essentially provides additional confirmation that upon cycling the resistance of this interface increases until the interface becomes disconnected during one particular switching cycle (as apparent from the $R_{\text {on }}$ jump in Fig. 5 for CELL AAO and as corroborated by Fig. 6).

In our view, to describe the features that lead to huge resistivity changes, we have to consider also the concept of conductive filaments as in the case of CELL THIN. However, in this case, a comparably thinner and shorter conductive filament over the ionic conductor is built in the period I shown in Fig. 1a. Nevertheless, it is conductive enough to dramatically decrease resistivity when built ( $R_{\text {on }}$ level), however easily dissolved in the reverse process with a strong impact on the resistivity ( $R_{\text {off }}$ level). This concept corresponds to the work of Wang et al. ${ }^{26}$ that assumes that the filament is connected with $\mathrm{Ag}^{+}$rich areas distributed in the ion conductive matrix and thus the volume of the ion conductor between electrodes directly influences the switching behavior. As apparent from Fig. 5, the $R_{\text {int }}$ value for CELL AAO is lower than any other value $R_{\text {off }}$ recorded afterwards. This can be explained by combination of dissolution of the $\mathrm{Ag}$ filament, or lowering the $\mathrm{Ag}$ content in non-stoichiometric $\mathrm{Ag}_{x} \mathrm{AsS}_{2}$ at the $\mathrm{Ag}-\mathrm{Ag}_{x} \mathrm{AsS}_{2}$ interface upon cycling. The continuous migration of $\mathrm{Ag}$ from nanowires towards the $\mathrm{Al}$ cathode through the ionic electrode results in slow depletion of $\mathrm{Ag}$ from $\mathrm{Ag}$ nanowires. $\mathrm{Ag}$-depletion of the $\mathrm{Ag}$ nanowire continuously increases with each new cycle, till contact is lost (as shown in Fig. 6) and the cell loses its function. Although the Ag-depletion is currently limiting the number of switching cycles that this type of cell is able to achieve, the magnitude of the resistance difference during the switching opens new pathways for an efficient data storage, or large data quantity.

We believe that by careful optimization, the number of achievable cycles could be strongly increased. We foresee several possibilities for a considerable increase of cycle numbers. The easiest option appears to increase the $\mathrm{Ag}$ content by deposition of a thin $\mathrm{Ag}$ film onto Ag-filled AAO, before depositing the ionic conductor. This Ag layer should have such thickness to become entirely dissolved in the $\mathrm{Ag}_{x} \mathrm{AsS}_{2}$ during the photodiffusion process. As a result, $\mathrm{Ag}_{x} \mathrm{AsS}_{2}$ would become $\mathrm{Ag}$ enriched (compared to situation without an additional $\mathrm{Ag}$ layer) and less Ag from nanowires would be consumed for the switching. Thus the number of achievable switching cycles 
would increase, potentially without any loss in the field focusing that helps us to maintain such a distinct resistance change between switching. So we could obtain an ideal compromise between the lifetime and the resistivity difference. It is noteworthy that adding an additional Pt layer in the NiO-based resistive switching cell with a positive effect on the switching has been reported recently. ${ }^{\mathbf{4 1}}$ Another possibility could be to improve the electrical contact between $\mathrm{Ag}$ nanowires and the chalcogenide ionic conductor by using an even smoother and cleaner Ag-filled AAO surface, prepared by optimized polishing and/or ion milling. It is possible that not all nanowires are electrically connected to the ionic conductor. Eventually, optimized AAO membranes for Ag-filling could be used. This will be a subject of further work.

\section{Conclusions}

We demonstrated comparably advanced design of resistive switching memory cells based on the Ag-filled porous anodic alumina membrane. The cells were prepared by low-cost combination of electrodeposition (filling of $\mathrm{Ag}$ into the membrane) and the thermal evaporation technique (to deposit layers of an ionic conductor $\mathrm{Ag}_{x} \mathrm{AsS}_{2}$ and $\mathrm{Al}$ contacts). The vital effect of a cell consisting of Ag-filled $\mathrm{AAO}$ and an ionic conductor $\mathrm{Ag}_{x} \mathrm{AsS}_{2}$ on the switching behavior was confirmed by direct comparison of samples with and without an Ag-filled AAO electrode. We achieved differences of 6 orders of magnitude in the resistivities between "on" and "off" states. Considering the great tuneability of dimensions of the AAO membrane and the possibility to use a whole range of ionic conductors, we believe that the presented approach could be explored and optimized for many other novel types of resistive switching memories based on the ionic effect.

\section{Acknowledgements}

Authors thank for financial support to project CZ.1.07./2.3.00/ 20.0254 "ReAdMat - Research Team for Advanced Non-crystalline Materials" co-financed by the European Social Fund and State Budget of the Czech Republic. We want to sincerely thank Prof. Keiji Tanaka for a fruitful discussion.

\section{Notes and references}

1 International Technology Roadmap for Semiconductors: Overall Roadmap Technology Characteristics, Semiconductor Industry Association, Incheon, Korea, 2011, available from http://public.itrs.net/.

2 C. Chappert, A. Fert and F. N. Van Dau, Nat. Mater., 2007, 6, 813-823.

3 M. D. Ventra and Y. V. Pershin, Mater. Today, 2011, 14, 584591.

4 R. Waser and M. Aono, Nat. Mater., 2007, 6, 833-840.

5 J. J. Yang, D. B. Strukov and D. R. Stewart, Nat. Nanotechnol., 2013, 8, 13-24.
6 T. Hasegawa, T. Ohno, K. Terabe, T. Tsuruoka, T. Nakazama, J. K. Gimyewski and M. Aono, Adv. Mater., 2010, 22, 18311834.

7 M. N. Kozicki, M. Mitkova, M. Park, M. Balakrishnan and C. Gopalan, Superlattices Microstruct., 2003, 34, 459-465.

8 M. N. Kozicki, M. Park and M. Mitkova, IEEE Trans. Nanotechnol., 2005, 4, 331-338.

9 C. Schindler, M. Meier, R. Waser and M. N. Kozicki, Nonvolatile Memory Technology Symposium, 2007, vol. 82-85, pp. 10-13.

10 R. Yang, K. Terabe, T. Tsuruoka, T. Hasegawa and M. Aono, Appl. Phys. Lett., 2012, 100, 231603.

11 C. Schindler, I. Valov and R. Waser, Phys. Chem. Chem. Phys., 2009, 11, 5974-5979.

12 X. B. Yan, J. Yin, H. X. Guo, Y. Su, B. Xu, H. T. Li, D. W. Yan, Y. D. Xia and Z. G. Liu, J. Appl. Phys., 2009, 106, 054501.

13 J. van den Hurk, I. Valov and R. Waser, Thin Solid Films, 2013, 527, 299-302.

14 M. Meier, S. Gilles, R. Rosezin, C. Schindler, S. Trellenkamp, A. Rüdiger, D. Mayer, C. Kügeler and R. Waser, Microelectron. Eng., 2009, 86, 1060-1062.

15 K. Nielsch, F. Müller, A.-P. Li and U. Gösele, Adv. Mater., 2000, 12, 582-586.

16 J. M. Baik, M. Schierhorn and M. Moskovits, J. Phys. Chem. C., 2008, 112, 2252-2255.

17 T.-H. Fang, T. H. Wang and S.-H. Kang, J. Mater. Sci., 2009, 44, 1588-1593.

18 W.-B. Zhao, J.-J. Zhu and H.-Y. Chen, Scr. Mater., 2004, 50, 1169-1173.

19 W.-C. Yoo and J.-K. Lee, Adv. Mater., 2004, 16, 1097-1101.

20 S.-H. Lyu and J.-S. Lee, J. Mater. Chem., 2012, 22, 1852-1861.

21 C. Liang, K. Terabe, T. Hasegawa, R. Negishi, T. Tamura and M. Aono, Small, 2005, 1, 971-975.

22 C. Liang, K. Terabe, T. Hasegawa and M. Aono, Nanotechnology, 2007, 18, 485202.

23 D. Perego, S. Franz, M. Bestetti, L. Cattaneo, S. Brivio, G. Tallarida and S. Spiga, Nanotechnology, 2013, 24, 045302.

24 D. Ielmini, C. Cagli, F. Nardi and Y. Zhang, J. Phys. D: Appl. Phys., 2013, 46, 074006.

25 T. Wagner, S. O. Kasap, Mir. Vlcek, M. Frumar, P. Nesladek and Mil. Vlcek, Appl. Surf. Sci., 2001, 175-176, 117-122.

26 F. Wang, W. P. Dunn, M. Jain, C. De Leo and N. Vickers, Solid-State Electron., 2011, 61, 33-37.

27 G. Sauer, G. Brehm, S. Schneider, K. Nielsch, R. B. Wehrspohn, J. Choi, H. Hofmeister and U. Gösele, J. Appl. Phys., 2002, 91, 3243-3247.

28 S. Stehlik, J. Kolar, M. Bartos, M. Vlcek, M. Frumar, V. Zima and T. Wagner, Solid State Ionics, 2010, 181, 1625-1630.

29 S. Wu, T. Tsuruoka, K. Terabe, T. Hasegawa, J. P. Hill, K. Ariga and M. Aono, Adv. Funct. Mater., 2011, 21, 93-99.

30 I. Valov, R. Waser, J. R. Jameson and M. N. Kozicki, Nanotechnology, 2011, 22, 254003.

31 K. Terabe, T. Hasegawa, T. Nakayama and M. Aono, Nature, 2005, 433, 47-50.

32 A. B. Seddon, J. Non-Cryst. Solids, 1995, 184, 44-50.

33 S. N. Yannopoulos, F. Kyriazis and I. P. Chochliouros, Opt. Lett., 2011, 36, 534-536. 
34 J. Orava, T. Wagner, M. Krbal, T. Kohoutek, Mil. Vlcek and M. Frumar, J. Non-Cryst. Solids, 2006, 352, 16371640.

35 S. Stehlik, J. Kolar, H. Haneda, I. Sakaguchi, M. Frumar and T. Wagner, Int. J. Appl. Glass Sci., 2011, 2, 301-307.

36 M. Frumar and T. Wagner, Curr. Opin. Solid State Mater. Sci., 2003, 7, 117-126.

37 X. Guo, C. Scindler, S. Menzel and R. Waser, Appl. Phys. Lett., 2007, 91, 133513.
38 M. Ohto, M. Itoh and K. Tanaka, J. Appl. Phys., 1995, 77, 1034-1039.

39 Q. Liu, S. Long, W. Wang, S. Tanachutiwat, Y. Li, Q. Wang, M. Zhang, Z. Huo, J. Chen and M. Liu, IEEE Electron Device Lett., 2010, 31, 1299-1301.

40 Z. Q. Wang, H. Y. Xu, L. Zhang, X. H. Li, J. G. Ma, X. T. Zhang and Y. C. Liu, Nanoscale, 2013, 5, 4490-4494.

41 Y.-C. Huang, P.-Y. Chen, T.-S. Chin, R.-S. Liu, C.-Y. Huang and C.-H. Lai, Appl. Phys. Lett., 2012, 101, 153106. 